\title{
Statin Use and Prostate Cancer Aggressiveness: Results from the Population-Based North Carolina-Louisiana Prostate Cancer Project
}

Emma H. Allott ${ }^{1,2}$, Laura Farnan ${ }^{2}$, Susan E. Steck ${ }^{3}$, Lenore Arab ${ }^{4}$, L. Joseph Su ${ }^{5}$, Merle Mishel ${ }^{6}$, Elizabeth T.H. Fontham ${ }^{7}$, James L. Mohler ${ }^{2,8}$, and Jeannette T. Bensen ${ }^{1,2}$

\section{Abstract}

Background: Although statin use has been associated with reduced prostate cancer aggressiveness, the impact of race and patient characteristics on this association is not well understood. We examined the association between statin use and prostate cancer aggressiveness in Caucasians (CA) and African Americans (AA) and explored effect modification by health-seeking behaviors associated with statin use.

Methods: Of 1,930 cases from The North Carolina-Louisiana Prostate Cancer Project, 344 (18\%) were classified as aggressive based on clinical criteria. Utilizing nonaggressive cases as referent, logistic regression was used to examine the association between statin use and prostate cancer aggressiveness, overall and stratified by race. Smoking and prostate cancer screening were examined as effect modifiers of this association.

Results: There was an inverse association between statin use and prostate cancer aggressiveness [OR, 0.74; 95\% confidence interval (CI), 0.56-0.96], with comparable effect estimates in both races. Although not statistically significant, statin use was associated with reduced ORs for aggressive prostate cancer in never-screened men (OR, 0.79; 95\% CI, 0.45-1.39), men screened at low/recommended frequency ( $\leq$ once/year; OR, $0.66 ; 95 \% \mathrm{CI}, 0.41-1.06)$, and men screened at high frequency (>once/year; OR, 0.78; 95\% CI, 0.53-1.15). Inverse associations between statins and aggressive prostate cancer were strongest in never smokers (OR, 0.42; 95\% CI, 0.25-0.72), attenuated in former smokers (OR, 0.84; 95\% CI, 0.59-1.19), and absent in current smokers (OR, 1.36; 95\% CI, 0.70-2.64).

Conclusions: Statin use was associated with reduced prostate cancer aggressiveness in CA and AAs, with strongest inverse associations in nonsmokers.

Impact: Health-seeking behaviors associated with statin use should be considered when examining the impact of statins on prostate cancer aggressiveness. Cancer Epidemiol Biomarkers Prev; 25(4); 670-7. (2016 AACR.

\section{Introduction}

Prostate cancer is the most commonly diagnosed noncutaneous cancer type in U.S. males and the second most common cause of male cancer-related deaths (1). Aggressive disease characteristics at diagnosis, defined by biopsy Gleason

'Department of Epidemiology, University of North Carolina at Chape Hill, Chapel Hill, North Carolina. ${ }^{2}$ Lineberger Comprehensive Cancer Center, University of North Carolina at Chapel Hill, Chapel Hill, North Carolina. ${ }^{3}$ Department of Epidemiology and Biostatistics, University of South Carolina, Columbia, South Carolina. ${ }^{4}$ David Geffen School of Medicine, University of California Los Angeles, Los Angeles, California. ${ }^{5}$ Winthrop P. Rockefeller Cancer Institute and College of Public Health, University of Arkansas for Medical Sciences, Little Rock, Arkansas. ${ }^{6}$ School of Nursing, University of North Carolina at Chapel Hill, Chapel Hill, North Carolina. ${ }^{7} \mathrm{~S} c h o o l$ of Public Health, Louisiana State University Health Sciences Center, New Orleans, Louisiana. ${ }^{8}$ Department of Urology, Roswell Park Cancer Institute, Buffalo, New York.

Note: Dr. Merle Mishel was not available to confirm coauthorship, but the corresponding author, Dr. Jeannette Bensen, affirms that Dr. Mishel contributed to the paper and thus confirms Dr. Mishel's coauthorship status.

Note: Supplementary data for this article are available at Cancer Epidemiology, Biomarkers \& Prevention Online (http://cebp.aacrjournals.org/).

Corresponding Author: Jeannette T. Bensen, University of North Carolina at Chapel Hill, 135 Dauer Dr., CB\#7400, Chapel Hill, NC 27599. Phone: 919-843-1017; Fax: 919-843-2439; E-mail: jeannette_bensen@med.unc.edu

doi: 10.1158/1055-9965.EPI-15-0631

(c2016 American Association for Cancer Research. sum, clinical stage, and PSA level, are associated with increased prostate cancer-specific mortality (2). As such, there is a need to identify factors which may impact prostate cancer aggressiveness.

Statins, a class of cholesterol-lowering drugs, are used by approximately one in every four adult males in the U.S. population (3). Although statin use is not associated with overall prostate cancer incidence (4-7), two meta-analyses have reported an inverse association between statin use and the risk of aggressive prostate cancer $(5,8)$. However, many of the studies contributing to these meta-analyses were limited by incomplete assessment of type and dose of statin and the use of other cholesterol-lowering drugs, as well as patient characteristics, including dietary cholesterol and saturated fat intake, smoking status, and prostate cancer screening history. In addition, these prior studies were conducted in predominantly Caucasian (CA) populations, and therefore the impact of race on these associations is unknown.

Using the population-based North Carolina-Louisiana Prostate Cancer Project (PCaP), we examined associations between statin use, dose and type, and prostate cancer aggressiveness, overall and stratified by race. We explored prostate cancer screening frequency as both a confounder and an effect modifier of the association between statin use and prostate cancer aggressiveness. In addition, we tested whether associations differed by smoking status, given that smoking is a known modifier of serum lipid levels. 


\section{Materials and Methods}

\section{Study population}

The PCaP is a population-based, case-only study of incident prostate cancer in two southern U.S. states (NC and LA; ref. 9). Men with the first diagnosis of histologically confirmed prostate cancer on or after July 1,2004, were eligible to participate in PCaP if they were 40 to 79 years of age at diagnosis, could complete the study interview in English, did not live in an institution or nursing home, and were not cognitively impaired. Eligibility criteria also required men to self-identify as either African American/black (AA) or CA/white in response to an open-ended question "what is your race?" Recruitment ended in October 2007 in NC and in July 2009 in LA. Research protocols were approved by the Institutional review boards at the University of North Carolina (Chapel Hill, NC), Louisiana State University Health Services Center, and Department of Defense CaP Research Program.

\section{Exposure assessment}

PCaP nurses administered a series of structured questionnaires that included baseline characteristics, prostate cancer screening history, and diet and medications during an in-home visit conducted approximately 3 months after diagnosis (9). Prostate cancer screening was defined as having received more than one PSA test and/or digital rectal exam (DRE) prior to 12 months before diagnosis. Among screened men, screening frequency was calculated as the total number of PSA or DRE in the patient's lifetime divided by the number of years since the first PSA or DRE. Screening was then classified as a 3-level variable (never screened, $\leq 1$ PSA and/or DRE per year, $>1$ PSA and/or DRE per year). We also generated this variable based upon PSA tests only, without considering DRE. Screening frequency cut-off points were selected on the basis of prostate cancer screening guidelines in place at the time of $\mathrm{PCaP}$ recruitment, which recommended annual screening. Screened men reporting $<1$ screening test per year were included with screened men reporting the recommended 1 test per year, given insufficient numbers to separate these two categories. The dietary assessment instrument was a modified version of the validated NCI Diet History Questionnaire (DHQ; refs. 10, 11) to which numerous Southern U.S. foods were added. Men were asked to report dietary intake during the 12 -month period prior to prostate cancer diagnosis. Dietary cholesterol intake (mg/day) and the average percentage of calories obtained from saturated fat were calculated using Diet* Calc Analysis Software [version 1.4.3, NCI (Bethesda, MD), Applied Research Program, November 2005]. Although no gold standard exists for validating calories from saturated fat and dietary cholesterol, a comparative validation study has been performed for the NCI DHQ, indicating that energy-adjusted correlations between multiple 24-hour recalls and DHQ estimates were 0.68 for saturated fat intake, 0.64 for dietary cholesterol, and 0.66 for total fat (11). Study participants gathered all prescription and nonprescription medications and supplements used in the 2-week period prior to interview and presented them to the nurse at the time of interview for documentation of current medication use, including type and dose. For this analysis, we abstracted statin use [atorvastatin (Lipitor, Caduet), simvastatin (Zocor, Vytorin), rosuvastatin (Crestor), lovastatin (Altoprev, Advicor), pravastatin (Pravachol), and fluvastatin (Lescol)] and nonstatin cholesterol-lowering drug use [niacin (Niaspan, Niacor), fibrate (gemfibrozil, Tricor), and ezetimibe (ezetimibe, Vytorin)]. Statin type was classified as hydrophilic (rosuvastatin and pravastatin) or lipophilic (atorvastatin, simvastatin, lovastatin, and fluvastatin; ref. 12). Statin dose was converted to a simvastatin dose equivalent, as described previously (13), and dichotomized as low/normal ( $\leq 20 \mathrm{mg}$ simvastatin dose equivalent) versus high dose ( $>20 \mathrm{mg}$ simvastatin dose equivalent). Data regarding the duration of statin use were not collected in this study.

\section{Outcome assessment}

Clinical stage, biopsy Gleason sum, and PSA at diagnosis were abstracted from medical records. Prostate cancer aggressiveness was defined using these three variables as follows: (i) high aggressive (Gleason sum $\geq 8$ or PSA $>20 \mathrm{ng} / \mathrm{mL}$ or Gleason sum $\geq 7$ and clinical stage T3-T4), (ii) low aggressive (Gleason sum $<7$ and clinical stage T1-T2 and PSA $<10 \mathrm{ng} / \mathrm{mL}$ ), and (iii) intermediate aggressive (all other cases), as described previously for PCaP (9). Our outcome of interest was high aggressive prostate cancer, and low/intermediate aggressive disease was utilized as the referent group for all analyses. Complete prostate cancer aggressiveness data were missing for 85 men, and these men were excluded from our analysis. We also excluded men who were missing body mass index (BMI; $n=21)$, smoking status $(n=2)$, and prostate cancer screening frequency $(n=220)$, resulting in 1,930 research subjects ( $n=1,012$ CA and $n=918$ AA) eligible for this analysis.

\section{Statistical analysis}

We examined differences in patient and tumor characteristics between CA and AA men, and between statin users and nonusers, using $\chi^{2}$ tests for categorical variables, Student $t$ tests for continuous, normally distributed variables, and rank sum tests for continuous nonnormally distributed variables.

Logistic regression was used to estimate crude (age-adjusted) and multivariable-adjusted ORs and 95\% confidence intervals (CI) for the association between statin use, dose and type, and prostate cancer aggressiveness (high vs. low/intermediate). Given that our outcome of high aggressive prostate cancer does not fit the rare disease assumption, ORs should not be interpreted as relative risk ratios. For multivariable analysis, we utilized a directed acyclic graph to select covariates and then performed backwards selection to build our final model, which included age (continuous), race (AA vs. CA), site (NC vs. LA), BMI (continuous and log transformed), cholesterol intake (continuous and log transformed), percent calories from saturated fat (continuous), smoking status (never, former, vs. current), and prostate cancer screening frequency (never, $\leq 1$ screening test per year, vs. $>1$ screening test per year). When examining associations between hydrophilic statins and prostate cancer aggressiveness, we excluded lipophilic statin users, and vice versa. We conducted a sensitivity analysis excluding men who used nonstatin cholesterol-lowering drugs $22 \%$ of statin users and $7 \%$ of statin nonusers). We also explored the effect of additionally adjusting models for education level (less than high school, high school graduate, vs. college graduate or some college), annual household income $(<\$ 20,000, \$ 20,000-$ $\$ 50,000, \$ 50,000-\$ 80,000$, vs. $>\$ 80,000$ ), and family history of prostate cancer in a first-degree relative (yes vs. no) in the subset of men for whom all of these data were available $(n=1,634)$. To examine smoking status and prostate cancer screening frequency as potential effect modifiers of the association between statin use and prostate cancer aggressiveness, we conducted stratified analysis by each of these health-seeking behaviors. We tested for interaction between smoking status and statin use for predicting prostate cancer aggressiveness by incorporating a cross-product 
Allott et al.

Table 1. Demographic and tumor characteristics of CA and AA prostate cancer cases in PCap

\begin{tabular}{|c|c|c|c|}
\hline & CA $(n=1,012)$ & AA $(n=918)$ & $P$ \\
\hline Age at diagnosis, mean (SD) & $64(8)$ & $62(8)$ & $<0.0001$ \\
\hline \multicolumn{4}{|l|}{ Site } \\
\hline North Carolina & $479(47)$ & $448(49)$ & \multirow[t]{2}{*}{0.519} \\
\hline Louisiana & $533(53)$ & $470(51)$ & \\
\hline \multicolumn{4}{|l|}{ Clinical stage } \\
\hline $\mathrm{T} 1$ & $559(55)$ & $509(55)$ & \multirow[t]{2}{*}{0.926} \\
\hline T2-T4 & $453(45)$ & $409(45)$ & \\
\hline PSA (ng/mL), median (IQR) & $5.2(4.1-7.5)$ & $6.2(4.5-10.7)$ & $<0.0001$ \\
\hline \multicolumn{4}{|l|}{ Biopsy Gleason sum } \\
\hline$\leq 3+4$ & $829(82)$ & $702(77)$ & \multirow[t]{2}{*}{0.006} \\
\hline$\geq 4+3$ & $180(18)$ & $208(23)$ & \\
\hline \multicolumn{4}{|l|}{ Aggressive prostate cancer } \\
\hline Low/intermediate & $861(85)$ & $725(79)$ & \multirow[t]{2}{*}{$<0.0001$} \\
\hline High & $151(15)$ & $193(21)$ & \\
\hline \multicolumn{4}{|l|}{ Prostate cancer screening frequency } \\
\hline Never & $116(11)$ & $328(36)$ & \multirow[t]{3}{*}{$<0.0001$} \\
\hline$\leq 1$ test per year & $386(38)$ & $377(75)$ & \\
\hline$>1$ test per year & $510(50)$ & $123(25)$ & \\
\hline \multicolumn{4}{|l|}{ Family history of prostate cancer (first-degree relative) ${ }^{a}$} \\
\hline No & $706(75)$ & $608(72)$ & \multirow[t]{2}{*}{0.122} \\
\hline Yes & $232(25)$ & $236(28)$ & \\
\hline \multicolumn{4}{|l|}{ Education } \\
\hline Less than high school & $94(9)$ & $265(29)$ & \multirow[t]{3}{*}{$<0.0001$} \\
\hline High school graduate & $212(21)$ & $258(28)$ & \\
\hline College graduate or some college & $706(70)$ & $394(43)$ & \\
\hline \multicolumn{4}{|l|}{ Income $^{\mathrm{b}}$} \\
\hline$<\$ 20,000$ & $91(10)$ & $273(32)$ & \multirow[t]{4}{*}{$<0.0001$} \\
\hline$\$ 20,000-\$ 50,000$ & $268(29)$ & $327(39)$ & \\
\hline$\$ 50,000-\$ 80,000$ & $229(25)$ & $137(16)$ & \\
\hline$>\$ 80,000$ & $337(36)$ & $105(12)$ & \\
\hline \multicolumn{4}{|l|}{ Smoking status } \\
\hline Never & $367(36)$ & $283(31)$ & \multirow[t]{3}{*}{$<0.0001$} \\
\hline Former & $551(54)$ & $441(48)$ & \\
\hline Current & $94(9)$ & $194(21)$ & \\
\hline \multicolumn{4}{|l|}{ BMI $\left(\mathrm{kg} / \mathrm{m}^{2}\right)$} \\
\hline$<30$ & $635(63)$ & $558(61)$ & \multirow[t]{2}{*}{0.375} \\
\hline$\geq 30$ & $377(37)$ & $360(39)$ & \\
\hline \multicolumn{4}{|l|}{ Charlson comorbidity index } \\
\hline 0 & $542(54)$ & $436(47)$ & 0.008 \\
\hline$\geq 1$ & $470(46)$ & $482(53)$ & \\
\hline Cardiovascular disease & & & \\
\hline No & $821(81)$ & $791(87)$ & 0.001 \\
\hline Yes & $187(19)$ & $118(13)$ & \\
\hline Diabetes & & & \\
\hline No & $842(84)$ & $674(74)$ & $<0.0001$ \\
\hline Yes & $166(16)$ & $239(26)$ & \\
\hline Dietary cholesterol intake (mg/day), median (IQR) & $262(180-355)$ & $302(206-439)$ & $<0.0001$ \\
\hline Percent saturated fat intake, mean (SD) & $11.2(2.7)$ & $10.0(2.6)$ & $<0.0001$ \\
\hline Nonstatin cholesterol-lowering drug use & & & \\
\hline None & $842(83)$ & $844(92)$ & $<0.0001$ \\
\hline Niacin/fibrate/ezetimibe & $170(17)$ & $74(8)$ & \\
\hline
\end{tabular}

Abbreviation: IQR, interquartile range.

${ }^{\mathrm{a}}$ Family history was missing for 74 CA and 74 AA men.

${ }^{\mathrm{b}}$ Income was missing for $12 \mathrm{CA}$ and $31 \mathrm{AA}$ men, whereas $75 \mathrm{CA}$ and $45 \mathrm{AA}$ men refused to answer this question.

term into the logistic regression model and calculating the global

$P$ value of the interaction term using the Wald test.

Statistical analyses were performed using Stata 13.1 (StataCorp). Statistical significance was two sided, with $P<0.05$.

\section{Results}

Characteristics of study participants by race

Incident prostate cancer cases in this study included 1,012 CAs ( $n=479$ from NC and $n=533$ from LA) and 918 AAs ( $n=448$ from NC and $n=470$ from LA; Table 1 ). As presented in Table 1,
AAs were younger at diagnosis than CAs (62 vs. 64 years of age) AAs had a higher median PSA level than CAs (6.2 vs. $5.2 \mathrm{ng} / \mathrm{mL}$ ) and were more likely to have a high biopsy Gleason sum $(\geq 4+3$ $23 \%$ vs. $18 \%)$ and aggressive prostate cancer (21\% vs. $15 \%)$.

AAs were less frequently screened for prostate cancer than CAs, with $36 \%$ of AAs and $11 \%$ of CAs never undergoing any prostate cancer screening (Table 1). In addition, AAs were less highly educated, reported a lower annual household income, and were more likely to be current smokers than CAs (21\% vs. $9 \%$; Table 1$)$. Despite lower prevalence of cardiovascular disease (13\% vs. $19 \%)$, AAs were more likely than CAs to have comorbid health 
conditions (Charlson index $\geq 1 ; 53 \%$ vs. $46 \%$ ), including diabetes ( $26 \%$ vs. $16 \%)$. Finally, AAs had higher dietary cholesterol intake (302 vs. $262 \mathrm{mg} /$ day), although the percentage of calories from saturated fat was higher in CAs (11.2\% vs. $10.0 \%$; Table 1$)$.

\section{Characteristics of study participants by statin use}

Of a total of 1,930 patients, $725(38 \%)$ were statin users at the time of interview, with the majority of statin users taking either simvastatin (38\%) or atorvastatin $(34 \%)$ and the remainder using rosuvastatin $(10 \%)$, pravastatin $(9 \%)$, lovastatin $(5 \%)$, or fluvastatin (2\%). There were no significant differences in statin type or dose by race (data not shown). As shown in Table 2, statin users were older than nonusers ( 65 vs. 62 years of age at diagnosis) and were more likely to be CA (56\% vs. $44 \%$ ). Although there were no significant differences in biopsy Gleason sum or clinical stage by statin use, statin users had lower median PSA level ( 5.3 vs.

Table 2. Demographic and tumor characteristics of statin users and nonusers in PCaP

\begin{tabular}{|c|c|c|c|}
\hline & $\begin{array}{c}\text { Statin nonusers } \\
(n=1,205)\end{array}$ & $\begin{array}{l}\text { Statin users } \\
(n=725)\end{array}$ & $\boldsymbol{P}$ \\
\hline Age at diagnosis, mean (SD) & $62(8)$ & $65(7)$ & $<0.0001$ \\
\hline \multicolumn{4}{|l|}{ Race } \\
\hline CA & $604(50)$ & $408(56)$ & \multirow[t]{2}{*}{0.009} \\
\hline AA & $601(50)$ & $317(44)$ & \\
\hline \multicolumn{4}{|l|}{ Site } \\
\hline North Carolina & $602(50)$ & $325(45)$ & \multirow[t]{2}{*}{0.029} \\
\hline Louisiana & $603(50)$ & $400(55)$ & \\
\hline \multicolumn{4}{|l|}{ Clinical stage } \\
\hline $\mathrm{T} 1$ & $679(56)$ & $389(54)$ & \multirow[t]{2}{*}{0.249} \\
\hline T2-T4 & $526(44)$ & $336(46)$ & \\
\hline PSA, median (IQR) & $5.8(4.3-9.8)$ & $5.3(4.1-7.6)$ & $<0.0001$ \\
\hline \multicolumn{4}{|l|}{ Biopsy Gleason sum } \\
\hline$\leq 3+4$ & $941(79)$ & $590(82)$ & \multirow[t]{2}{*}{0.101} \\
\hline$\geq 4+3$ & $256(21)$ & $132(18)$ & \\
\hline \multicolumn{4}{|l|}{ Aggressive prostate cancer } \\
\hline Low/intermediate & $972(81)$ & $614(85)$ & \multirow[t]{2}{*}{0.025} \\
\hline High & $233(19)$ & $111(15)$ & \\
\hline \multicolumn{4}{|l|}{ Prostate cancer screening frequency } \\
\hline Never & $278(23)$ & $82(11)$ & \multirow[t]{3}{*}{$<0.0001$} \\
\hline$\leq 1$ Test per year & $365(30)$ & $260(36)$ & \\
\hline$>1$ Test per year & $562(47)$ & $383(53)$ & \\
\hline \multicolumn{4}{|c|}{ Family history of prostate cancer (first-degree relative) ${ }^{a}$} \\
\hline No & $811(73)$ & $503(75)$ & \multirow[t]{2}{*}{0.214} \\
\hline Yes & $304(27)$ & $164(25)$ & \\
\hline \multicolumn{4}{|l|}{ Education } \\
\hline Less than high school & $234(19)$ & $125(17)$ & \multirow[t]{3}{*}{0.320} \\
\hline High school graduate & $299(25)$ & $171(24)$ & \\
\hline College graduate or some college & $672(56)$ & $428(59)$ & \\
\hline \multicolumn{4}{|l|}{ Income ${ }^{b}$} \\
\hline$<\$ 20,000$ & $238(22)$ & $126(19)$ & \multirow[t]{4}{*}{0.331} \\
\hline$\$ 20,000-\$ 50,000$ & $361(33)$ & $234(35)$ & \\
\hline$\$ 50,000-\$ 80,000$ & $221(20)$ & $145(22)$ & \\
\hline$>\$ 80,000$ & $284(25)$ & $158(24)$ & \\
\hline \multicolumn{4}{|l|}{ Smoking status } \\
\hline Never & 407 (34) & $243(34)$ & \multirow[t]{3}{*}{$<0.0001$} \\
\hline Former & $585(49)$ & $407(56)$ & \\
\hline Current & $213(18)$ & $75(10)$ & \\
\hline BMI $\left(\mathrm{kg} / \mathrm{m}^{2}\right)$ & & & \\
\hline$<30$ & $793(66)$ & $400(55)$ & $<0.0001$ \\
\hline$>30$ & $412(34)$ & $325(45)$ & \\
\hline Charlson comorbidity index & & & \\
\hline 0 & $697(58)$ & $281(39)$ & $<0.0001$ \\
\hline$\geq 1$ & $508(42)$ & $444(61)$ & \\
\hline Cardiovascular disease & & & \\
\hline No & $1,104(92)$ & $508(71)$ & $<0.0001$ \\
\hline Yes & $95(8)$ & $210(29)$ & \\
\hline Diabetes & & & \\
\hline No & $1,022(85)$ & $494(69)$ & $<0.0001$ \\
\hline Yes & $179(15)$ & $226(31)$ & \\
\hline Dietary cholesterol intake (mg/day) & $288(198-406)$ & $269(179-368)$ & 0.0001 \\
\hline Percent saturated fat intake, mean (SD) & $10.6(2.7)$ & $10.7(2.7)$ & 0.811 \\
\hline Nonstatin cholesterol-lowering drug use & & & \\
\hline None & $1,123(93)$ & $563(78)$ & $<0.0001$ \\
\hline Niacin/fibrate/ezetimibe & $82(7)$ & $162(22)$ & \\
\hline
\end{tabular}

Abbreviation: IQR, interquartile range.

${ }^{a}$ Family history missing for 90 statin nonusers and 58 statin users.

${ }^{\mathrm{b}}$ Income was missing for 27 statin nonusers and 16 statin users, whereas 74 statin nonusers and 46 statin users refused to answer this question. 
Table 3. Associations between statin use, dose and type, and prostate cancer aggressiveness, overall and stratified by race

\begin{tabular}{|c|c|c|c|c|c|c|c|c|c|}
\hline & \multicolumn{3}{|c|}{ All } & \multicolumn{3}{|c|}{ CA } & \multicolumn{3}{|c|}{ AA } \\
\hline & $\begin{array}{c}n, \text { cases } \\
\text { (aggressive) }\end{array}$ & $\mathrm{OR}^{\mathrm{a}}(95 \% \mathrm{Cl})$ & $\mathrm{OR}^{\mathrm{b}}(95 \% \mathrm{Cl})$ & $\begin{array}{c}n, \text { cases } \\
\text { (aggressive) }\end{array}$ & $\mathrm{OR}^{\mathrm{a}}(95 \% \mathrm{Cl})$ & $\mathrm{OR}^{\mathrm{b}}(95 \% \mathrm{CI})$ & $\begin{array}{c}n, \text { cases } \\
\text { (aggressive) }\end{array}$ & $\mathrm{OR}^{\mathrm{a}}(95 \% \mathrm{Cl})$ & $\mathrm{OR}^{\mathrm{b}}(95 \% \mathrm{Cl})$ \\
\hline \multicolumn{10}{|l|}{ Statin use } \\
\hline No use & 1,205 (233) & 1.00 (ref) & 1.00 (ref) & 604 (99) & 1.00 (ref) & 1.00 (ref) & $601(134)$ & 1.00 (ref) & 1.00 (ref) \\
\hline Use & 725 (111) & $\begin{array}{c}0.68 \\
(0.53-0.87)\end{array}$ & $\begin{array}{c}0.74 \\
(0.56-0.96)\end{array}$ & $408(52)$ & $\begin{array}{c}0.65 \\
(0.45-0.93)\end{array}$ & $\begin{array}{c}0.64 \\
(0.44-0.95)\end{array}$ & 317 (59) & $\begin{array}{c}0.73 \\
(0.51-1.03)\end{array}$ & $\begin{array}{c}0.84 \\
(0.58-1.21)\end{array}$ \\
\hline \multicolumn{10}{|l|}{ Statin dose $e^{c}$} \\
\hline No use & $1,205(233)$ & 1.00 (ref) & 1.00 (ref) & 604 (99) & 1.00 (ref) & 1.00 (ref) & $601(134)$ & 1.00 (ref) & 1.00 (ref) \\
\hline Low/normal & $306(45)$ & $\begin{array}{c}0.65 \\
(0.45-0.92)\end{array}$ & $\begin{array}{c}0.70 \\
(0.49-1.01)\end{array}$ & $180(24)$ & $\begin{array}{c}0.68 \\
(0.42-1.11)\end{array}$ & $\begin{array}{c}0.66 \\
(0.40-1.09)\end{array}$ & $126(21)$ & $\begin{array}{c}0.64 \\
(0.38-1.06)\end{array}$ & $\begin{array}{c}0.74 \\
(0.43-1.26)\end{array}$ \\
\hline High & $419(66)$ & $\begin{array}{c}0.70 \\
(0.52-0.95)\end{array}$ & $\begin{array}{c}0.76 \\
(0.55-1.04)\end{array}$ & $228(28)$ & $\begin{array}{c}0.62 \\
(0.39-0.98)\end{array}$ & $\begin{array}{c}0.63 \\
(0.39-1.01)\end{array}$ & $191(38)$ & $\begin{array}{c}0.79 \\
(0.53-1.19)\end{array}$ & $\begin{array}{c}0.90 \\
(0.59-1.38)\end{array}$ \\
\hline \multicolumn{10}{|l|}{ Statin type ${ }^{d}$} \\
\hline No use & $1,205(233)$ & 1.00 (ref) & 1.00 (ref) & 604 (99) & 1.00 (ref) & 1.00 (ref) & 601 (134) & 1.00 (ref) & 1.00 (ref) \\
\hline Hydrophilic & $132(16)$ & $\begin{array}{c}0.53 \\
(0.31-0.91)\end{array}$ & $\begin{array}{c}0.56 \\
(0.32-0.99)\end{array}$ & $81(8)$ & $\begin{array}{c}0.48 \\
(0.22-1.05)\end{array}$ & $\begin{array}{c}0.45 \\
(0.20-1.00)\end{array}$ & $51(8)$ & $\begin{array}{c}0.62 \\
(0.29-1.36)\end{array}$ & $\begin{array}{c}0.72 \\
(0.32-1.62)\end{array}$ \\
\hline Lipophilic & $591(94)$ & $0.71(0.54-0.92)$ & $\begin{array}{c}0.77 \\
(0.58-1.02)\end{array}$ & $327(44)$ & $\begin{array}{c}0.69 \\
(0.46-1.02)\end{array}$ & $\begin{array}{c}0.69 \\
(0.46-1.04)\end{array}$ & $264(50)$ & $\begin{array}{c}0.74 \\
(0.51-1.07)\end{array}$ & $\begin{array}{c}0.86 \\
(0.58-1.27)\end{array}$ \\
\hline
\end{tabular}

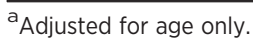

${ }^{\mathrm{b}}$ Adjusted for age, race (except for analyses stratified by race), site, BMI, cholesterol intake, percent saturated fat intake, smoking status, and prostate cancer screening frequency.

${ }^{c}$ Low/normal dose $\leq 20 \mathrm{mg}$ simvastatin or equivalent; high dose $>20 \mathrm{mg}$ simvastatin or equivalent.

${ }^{d}$ Hydrophilic = rosuvastatin and pravastatin; lipophilic = atorvastatin, simvastatin, lovastatin, and fluvastatin.

$5.8 \mathrm{ng} / \mathrm{mL}$ ) and a lower frequency of aggressive prostate cancer (15\% vs. $19 \%)$.

Statin users were more frequently screened for prostate cancer, relative to nonusers (Table 2). Statin users had similar level of education and annual household income as nonusers but were less likely to be current smokers ( $10 \%$ vs. $18 \%$; Table 2 ). Relative to nonusers, statin users were more likely to be obese $(44 \%$ vs. $34 \%$ ) and have a comorbid condition (Charlson index $\geq 1 ; 61 \%$ vs. $42 \%$ ), including diabetes ( $31 \%$ vs. $15 \%$ ) and cardiovascular disease ( $29 \%$ vs. $8 \%$; Table 2 ). However, statin users had lower dietary cholesterol intake ( 269 vs. $288 \mathrm{mg} /$ day), although there was no difference in saturated fat intake between statin users and nonusers.

We also examined differences in statin users versus nonusers stratified by race and found that these aforementioned differences in tumor and patient characteristics between statin users and nonusers were observed in both CAs and AAs (Supplementary Table S1).

\section{Associations between statin use and prostate cancer aggressiveness}

Of $344(18 \%)$ incident cases of high aggressive prostate cancer, $111(32 \%)$ occurred in statin users and 233 (68\%) occurred in nonusers. For comparison, of 1,586 (82\%) incident cases of nonaggressive prostate cancer, 614 (39\%) occurred in statin users and $972(61 \%)$ occurred in nonusers. Utilizing nonaggressive cases as the referent group, and adjusting for potential confounders, statin use was associated with a significantly reduced OR for aggressive prostate cancer (OR, 0.74; 95\% CI, 0.56-0.96; Table 3), with similar effect estimates in CAs (OR, 0.64; 95\% CI, 0.44-0.95) and AAs (OR, 0.84; 95\% CI, 0.58-1.21), although the association in AAs was not statistically significant. We observed similar inverse associations in men taking a low/normal statin dose (OR, 0.70; 95\% CI, 0.49-1.01) and in men taking a high statin dose (OR, 0.76; 95\% CI, 0.58-1.04), with no evidence for a dose-response relationship, although small numbers of men in each dose category may limit our power to detect such a relationship.
Finally, although there was a suggestion of a stronger protective effect with hydrophilic relative to lipophilic statins (OR, 0.56; $95 \% \mathrm{CI}, 0.32-0.99$ and OR, 0.77; 95\% CI, 0.58-1.02, respectively), these estimates were somewhat imprecise due to low numbers of men using hydrophilic statins and should be interpreted cautiously. Excluding men using nonstatin cholesterol-lowering drugs or additionally adjusting our models for education level, annual household income, and family history of prostate cancer did not substantially alter our findings (Supplementary Tables S2 and S3, respectively).

Impact of health-seeking behaviors on the association between statin use and prostate cancer aggressiveness

To explore possible prostate cancer screening-related detection biases, we examined prostate cancer screening frequency as an effect modifier of the association between statin use and prostate cancer aggressiveness. We observed nonstatistically significant inverse associations between statin use and prostate cancer aggressiveness in men who had never undergone PSA or DRE screening (OR, 0.79; 95\% CI, 0.45-1.39), in men who were screened annually or less frequently (OR, 0.66; 95\% CI, $0.41-1.06)$, and in men who were screened more frequently than once a year (OR, 0.78; 95\% CI, 0.53-1.15; Table 4), although these findings should be interpreted cautiously, given relatively small numbers in each screening category. We observed similar findings when we classified prostate cancer screening as PSA tests only, without considering DRE (data not shown).

Smoking increases low-density and total cholesterol levels and decreases high-density cholesterol levels $(14,15)$, potentially offsetting the cholesterol-lowering effect of statin use. We found no association between statin use and prostate cancer aggressiveness in current smokers (OR, 1.36; 95\% CI, 0.70-2.64). In contrast, there was a strong inverse association between statin use and prostate cancer aggressiveness in never smokers (OR, 0.42; $95 \%$ CI, 0.25-0.72), with an attenuated protective effect in former smokers (OR, 0.84; 95\% CI, 0.59-1.19; Table 5) and a significant 
Table 4. Associations between statin use and prostate cancer aggressiveness, stratified by prostate cancer screening frequency

\begin{tabular}{|c|c|c|c|}
\hline & $\begin{array}{c}n, \text { cases } \\
\text { (aggressive) }\end{array}$ & $\mathrm{OR}^{\mathrm{a}}(95 \% \mathrm{Cl})$ & $\mathrm{OR}^{\mathrm{b}}(95 \% \mathrm{Cl})$ \\
\hline \multicolumn{4}{|l|}{ Never screened } \\
\hline Statin nonusers & $278(87)$ & 1.00 (ref) & 1.00 (ref) \\
\hline Statin users & $82(23)$ & $0.81(0.47-1.41)$ & $0.79(0.45-1.39)$ \\
\hline \multicolumn{4}{|c|}{$\leq 1$ Screening tests per year } \\
\hline Statin nonusers & $365(61)$ & 1.00 (ref) & 1.00 (ref) \\
\hline Statin users & $260(35)$ & $0.67(0.42-1.07)$ & $0.66(0.41-1.06)$ \\
\hline \multicolumn{4}{|c|}{$>1$ Screening tests per year } \\
\hline Statin nonusers & $562(85)$ & 1.00 (ref) & 1.00 (ref) \\
\hline Statin users & $383(53)$ & $0.79(0.54-1.15)$ & $0.78(0.53-1.15)$ \\
\hline
\end{tabular}

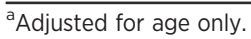

${ }^{\mathrm{b}}$ Adjusted for age, race, site, BMI, cholesterol intake, percent saturated fat intake, and smoking status.

interaction between smoking status and statin use in predicting prostate cancer aggressiveness (Wald test; $P=0.0008$ ).

\section{Discussion}

Using data from the population-based PCaP study, we report an inverse association between statin use and prostate cancer aggressiveness. We observed a similar magnitude of association between statin use and prostate cancer aggressiveness in both CAs and AAs; findings were supported by a prior study, which found that associations between statin use and risk of high-grade prostate cancer did not vary by race (16). In contrast to prior cohort and case-control analyses of statin use and prostate cancer risk using prostate cancer-free men as the referent group $(5,8,16)$, the case-only design of this study examined the impact of statin use on disease aggressiveness among men diagnosed with prostate cancer. However, our results are in agreement with approximately $20 \%$ to $25 \%$ reduced risk of aggressive prostate cancer in statin users, relative to nonusers reported by two meta-analyses $(5,8)$. As such, our findings strengthen existing rationale to explore a role for statins in aggressive prostate cancer prevention.

One important consideration when studying the impact of statin use on prostate cancer aggressiveness is that detection bias arising from higher rates of prostate cancer screening in statin users could produce an inverse association with aggressive disease, irrespective of a causal relationship $(17,18)$. In this study, we found that adjusting our models for prostate cancer screening frequency did not substantially impact our estimates. Furthermore, analyses stratified by screening frequency showed a similar magnitude of inverse association between statin use and prostate

Table 5. Associations between statin use and prostate cancer aggressiveness, stratified by smoking status

\begin{tabular}{lccc}
\hline & $\begin{array}{c}\mathbf{n}, \text { cases } \\
\text { (aggressive) }\end{array}$ & $\mathbf{O R}^{\mathbf{a}} \mathbf{( 9 5 \% ~ \mathbf { ~ I } )}$ & $\mathbf{O R}^{\mathbf{b}} \mathbf{( 9 5 \% ~ C l )}$ \\
\hline Never smokers & & & \\
$\quad$ Statin nonusers & $407(69)$ & $1.00($ ref $)$ & $1.00($ ref $)$ \\
$\quad$ Statin users & $243(24)$ & $0.43(0.26-0.72)$ & $0.42(0.25-0.72)$ \\
Former smokers & & & \\
$\quad$ Statin nonusers & $585(109)$ & $1.00($ ref $)$ & $1.00($ ref $)$ \\
$\quad$ Statin users & $407(67)$ & $0.78(0.56-1.10)$ & $0.84(0.59-1.19)$ \\
Current smokers & & & \\
$\quad$ Statin nonusers & $213(55)$ & $1.00($ ref $)$ & $1.00($ ref $)$ \\
$\quad$ Statin users & $75(20)$ & $1.04(0.57-1.90)$ & $1.36(0.70-2.64)$ \\
\hline
\end{tabular}

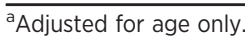

${ }^{\mathrm{b}}$ Adjusted for age, race, site, BMI, cholesterol intake, percent saturated fat intake, and prostate cancer screening frequency. cancer aggressiveness in men screened at high frequency (i.e., more than once a year), low, or recommended frequency (i.e., annually or less) and in unscreened men, suggesting that the association between statin use and prostate cancer aggressiveness cannot be completely explained by screening-related detection bias. In support of these findings, inverse associations between statin use and aggressive prostate cancer have been reported both in European populations with very low screening rates (19) and in U.S. populations with higher screening rates (20), although a secondary analysis of the Prostate Cancer Prevention Trial, wherein all participants were screened annually, reported no association between statin use and high-grade prostate cancer (21). Moreover, an analysis of simulated datasets with different PSA screening frequencies suggested that detection bias is unlikely to explain the association between statin use and reduced prostate cancer aggressiveness (18). Yet another factor to consider is that statin use lowers PSA levels by approximately $3 \%$ to $4 \%(22,23)$, potentially reducing the likelihood of prostate biopsy and aggressive prostate cancer diagnosis. Indeed, we observed that statin users had lower PSA levels than nonusers in this study. Randomized trials conducting PSA-independent biopsies at regular intervals may circumvent this potential source of detection bias, and secondary analysis of one such trial reported a null association between statin use and high-grade prostate cancer (24). However, given that trial participants are selected based upon specific eligibility criteria and do not represent a population-based sample, findings from secondary analyses of trials $(21,24)$ may differ from findings reported by population-based studies $(19,20)$, including our own. As such, although the potential for screening-related detection biases should be considered, our findings, in addition to those from populations with different PSA screening frequencies (25), support a true association between statin use and prostate cancer aggressiveness.

In addition to differences in prostate cancer screening behaviors, characteristics of statin users differ from those of nonusers in a variety of ways. Data from this study show that obesity and diabetes, both associated with increased prostate cancer-specific mortality $(26,27)$, were more prevalent among statin users. On the other hand, we observed that statin use was associated with health-seeking behaviors, as indicated by the higher prevalence of prostate cancer screening, lower prevalence of smoking, and reduced dietary cholesterol intake, relative to nonusers. These health-seeking behaviors themselves have been associated with reduced risk of aggressive prostate cancer $(17,28-33)$, potentially giving rise to a "healthy-user" bias, whereby the association between statin use and prostate cancer aggressiveness could be explained by the health-seeking behaviors of statin users, and not statin use per se (34). In this analysis, we found that adjusting our models for these health-seeking behaviors did not substantially impact our estimates. However, analyses stratified on smoking status revealed a strong inverse association between statin use and prostate cancer aggressiveness in never smokers, a slightly attenuated effect in former smokers, and no association in current smokers. There may be multiple mechanisms contributing to the observed effect modification by smoking status, one of which may be the association between smoking and prostate cancer aggressiveness $(32,33)$. However, given that the impact of statins on prostate cancer may be mediated, at least in part, via their cholesterol-lowering properties (35), a smoking-related increase in cholesterol level $(14,15)$ could potentially offset the protective effect of statins on prostate cancer aggressiveness. If confirmed in 
future studies, these hypothesis-generating findings may add to the many established reasons to promote smoking cessation for direct and indirect reduction of a myriad of noncommunicable diseases.

Our findings should be considered in light of the strengths and limitations of this study. First, statin use was captured at the time of interview, with no information regarding the timing of statin initiation relative to prostate cancer diagnosis. However, given that the majority of our study population was interacting with the health care system prior to prostate cancer diagnosis $(75 \%$ of individuals had a history of PSA screening), it is likely that men indicated for statin therapy would have initiated statins before diagnosis. Indeed, a previous study reported similar prevalence of statin use before (29\%) and after (30\%) prostate cancer diagnosis, during a study period (1998-2009) which overlapped that of this study (2004-2009; ref. 36). Moreover, any potential misclassification of unexposed individuals (i.e., prediagnosis nonusers who initiated statin use after diagnosis) as exposed individuals (i.e., prediagnosis statin users) would likely bias our estimates towards the null. As such, our study may have underestimated the strength of the association between prediagnosis statin use and prostate cancer aggressiveness. In addition, although we did not have access to data for duration of use or adherence to statin therapy, the type and dose of statin and nonstatin cholesterol-lowering drugs were documented by a trained nurse, thus improving the accuracy of our exposure data. Second, although serum cholesterol measurements were unavailable, dietary cholesterol and saturated fat intake were available for all study participants, and saturated fat intake is an important determinant of serum cholesterol level (37). Third, we lacked sufficient numbers to separate men reporting annual prostate cancer screening from men reporting less-than-annual screening, and larger studies should explore associations between statins and prostate cancer aggressiveness within each of these categories. Fourth, our study had somewhat limited statistical power to conduct analyses stratified by healthseeking behaviors, and larger studies are required to further explore these hypotheses. Finally, observational studies examining the association between statin use and prostate cancer are susceptible to confounding by indication, given that statin use is not randomized. However, an important strength of this study is our comprehensive assessment of clinical and demographic characteristics, in addition to health-seeking behaviors of statin users and nonusers, and adjustment for these potential confounders in our analysis.

In summary, we report an inverse association between statin use and aggressive prostate cancer in both CAs and AAs. Differences in patient characteristics and health-seeking behaviors by statin use should be an important consideration for future observational studies of statin use and prostate cancer, although our

\section{References}

1. Siegel R, Ma J, Zou Z, Jemal A. Cancer statistics, 2014. CA Cancer J Clin 2014;64:9-29.

2. Daskivich TJ, Fan KH, Koyama T, Albertsen PC, Goodman M, Hamilton AS et al. Prediction of long-term other-cause mortality in men with early-stage prostate cancer: results from the Prostate Cancer Outcomes Study. Urology 2015;85:92-100.

3. Gu Q, Paulose-Ram R, Burt VL, Kit BK. Prescription cholesterol-lowering medication use in adults aged 40 and over: United States, 2003-2012. Hyattsville, MD: National Center for Health Statistics; 2014. findings suggest that the detection bias arising from higher rates of prostate cancer screening in statin users does not entirely explain the inverse association between statin use and prostate cancer aggressiveness. The stronger protective effect of statin use in nonsmokers requires confirmation in other studies but may add to existing rationale, supporting efforts to reduce smoking rates in the population. Statins are cost-effective and widely prescribed cholesterol-lowering drugs, and recent cholesterol-lowering guidelines (38) will likely further increase the prevalence of statin use. Given that cardiovascular disease and cancer are the two most common causes of mortality in the United States (39), with prostate cancer the second most common cause of cancer-related death in U.S. men (1), understanding the role of statins in aggressive prostate cancer prevention will have important public health impact.

\section{Disclosure of Potential Conflicts of Interest}

E.T.H. Fontham is a consultant/advisory board member for NCI. No potential conflicts of interest were disclosed by the other authors.

\section{Authors' Contributions}

Conception and design: E.H. Allott, L. Arab, L.J. Su, M. Mishel, E.T.H. Fontham, J.T. Bensen

Development of methodology: E.H. Allott, L. Arab, M. Mishel, J.T. Bensen

Acquisition of data (provided animals, acquired and managed patients, provided facilities, etc.): S.E. Steck, L. Arab, L.J. Su, E.T.H. Fontham, J.L. Mohler, J.T. Bensen

Analysis and interpretation of data (e.g., statistical analysis, biostatistics, computational analysis): E.H. Allott, L. Farnan, S.E. Steck, L. Arab, J.T. Bensen Writing, review, and/or revision of the manuscript: E.H. Allott, L. Farnan, S.E. Steck, L. Arab, L.J. Su, E.T.H. Fontham, J.L. Mohler, J.T. Bensen

Administrative, technical, or material support (i.e., reporting or organizing data, constructing databases): L. Farnan, J.L. Mohler

Study supervision: E.T.H. Fontham, J.L. Mohler, J.T. Bensen

\section{Acknowledgments}

The North Carolina-Louisiana Prostate Cancer Project (PCaP) is carried out as a collaborative study supported by the Department of Defense contract DAMD 17-03-2-0052. The authors thank the staff, advisory committees, and research subjects participating in the PCaP study for their important contributions.

\section{Grant Support}

This work was supported by the University Cancer Research Fund of North Carolina (to E.H. Allott).

The costs of publication of this article were defrayed in part by the payment of page charges. This article must therefore be hereby marked advertisement in accordance with 18 U.S.C. Section 1734 solely to indicate this fact.

Received June 11, 2015; revised October 6, 2015; accepted October 24, 2015; published OnlineFirst January 27, 2016. 
8. Bansal D, Undela K, D'Cruz S, Schifano F. Statin use and risk of prostate cancer: a meta-analysis of observational studies. PLoS One 2012;7: e46691.

9. Schroeder JC, Bensen JT, Su LJ, Mishel M, Ivanova A, Smith GJ, et al. The North Carolina-Louisiana Prostate Cancer Project (PCaP): methods and design of a multidisciplinary population-based cohort study of racial differences in prostate cancer outcomes. Prostate 2006;66:1162-76.

10. Thompson FE, Subar AF, Brown CC, Smith AF, Sharbaugh CO, Jobe JB, et al. Cognitive research enhances accuracy of food frequency questionnaire reports: results of an experimental validation study. J Am Diet Assoc 2002;102:212-25.

11. Subar AF, Thompson FE, Kipnis V, Midthune D, Hurwitz P, McNutt S, et al Comparative validation of the Block, Willett, and National Cancer Institute Food Frequency Questionnaires: The Eating at America's Table Study. Am J Epidemiol 2001;154:1089-99.

12. Roy M, Kung HJ, Ghosh PM. Statins and prostate cancer: role of cholesterol inhibition vs. prevention of small GTP-binding proteins. Am J Cancer Res 2011;1:542-61.

13. Hamilton RJ, Banez LL, Aronson WJ, Terris MK, Platz EA, Kane CJ, et al. Statin medication use and the risk of biochemical recurrence after radical prostatectomy: results from the Shared Equal Access Regional Cancer Hospital (SEARCH) Database. Cancer 2010;116:3389-98.

14. Freeman DJ, Griffin BA, Murray E, Lindsay GM, Gaffney D, Packard CJ, et al. Smoking and plasma lipoproteins in man: effects on low density lipoprotein cholesterol levels and high density lipoprotein subfraction distribution. Eur J Clin Invest 1993;23:630-40.

15. Wynder EL, Harris RE, Haley NJ. Population screening for plasma cholesterol: community-based results from Connecticut. Am Heart J 1989; $117: 649-56$.

16. Kantor ED, Lipworth L, Fowke JH, Giovannucci EL, Mucci LA, Signorello LB. Statin use and risk of prostate cancer: results from the Southern Community Cohort Study. Prostate 2015;75:1384-93.

17. Catalona WJ, Smith DS, Ratliff TL, Basler JW. Detection of organ-confined prostate cancer is increased through prostate-specific antigen-based screening. JAMA 1993;270:948-54.

18. Mondul AM, Caffo B, Platz EA. Minimal detection bias in the inverse association between statin drug use and advanced prostate cancer risk: a simulation study. Cancer Epidemiol 2011;35:e6-11.

19. Murtola TJ, Tammela TL, Lahtela J, Auvinen A. Cholesterol-lowering drugs and prostate cancer risk: a population-based case-control study. Cancer Epidemiol Biomarkers Prev 2007;16:2226-32.

20. Platz EA, Leitzmann MF, Visvanathan K, Rimm EB, Stampfer MJ, Willett $\mathrm{WC}$, et al. Statin drugs and risk of advanced prostate cancer. J Natl Cancer Inst 2006;98:1819-25.

21. Platz EA, Tangen CM, Goodman PJ, Till C, Parnes HL, Figg WD, et al. Statin drug use in not associated with prostate cancer risk in men who are regularly screened. J Urol 2014;192:379-84.

22. Hamilton RJ, Goldberg KC, Platz EA, Freedland SJ. The influence of statin medications on prostate -specific antigen levels. J Natl Cancer Inst 2008; 100:1511-8.

23. Chang SL, Harshman LC, Presti JC. Impact of common medications on serum total prostate-specific antigen levels: analysis of the National Health and Nutrition Examination Survey. J Clin Oncol 2010;28:3951-7.
24. Freedland SJ, Hamilton RJ, Gerber L, Banez LL, Moreira DM, Andriole GL, et al. Statin use and risk of prostate cancer and high-grade prostate cancer: results from the REDUCE study. Prostate Cancer Prostatic Dis 2013;16: 254-9.

25. Platz EA. Epidemiologic musing on statin drugs in the prevention of advanced prostate cancer. Cancer Epidemiol Biomarkers Prev 2007;16: 2175-80.

26. Cai H, Xu Z, Xu T, Yu B, Zou Q. Diabetes mellitus is associated with elevated risk of mortality amongst patients with prostate cancer: a meta-analysis of 11 cohort studies. Diabetes Metab Res Rev 2015;31:336-43.

27. Allott EH, Masko EM, Freedland SJ. Obesity and prostate cancer: weighing the evidence. Eur Urol 2013;63:800-9.

28. Mondul AM, Weinstein SJ, Virtamo J, Albanes D. Serum total and HDL cholesterol and risk of prostate cancer. Cancer Causes Control 2011;22: $1545-52$.

29. Platz EA, Till C, Goodman PJ, Parnes HL, Figg WD, Albanes D, et al. Men with low serum cholesterol have a lower risk of high-grade prostate cancer in the placebo arm of the prostate cancer prevention trial. Cancer Epidemiol Biomarkers Prev 2009;18:2807-13.

30. Shafique K, McLoone P, Qureshi K, Leung H, Hart C, Morrison DS. Cholesterol and the risk of grade-specific prostate cancer incidence: evidence from two large prospective cohort studies with up to 37 years' follow up. BMC Cancer 2012;12:25.

31. Farwell WR, D'Avolio LW, Scranton RE, Lawler EV, Gaziano JM. Statins and prostate cancer diagnosis and grade in a veterans population. J Natl Cancer Inst 2011;103:885-92.

32. $\mathrm{Zu} \mathrm{K}$, Giovannucci E. Smoking and aggressive prostate cancer: a review of the epidemiologic evidence. Cancer Causes Control 2009;20: 1799-810.

33. Ho T, Howard LE, Vidal AC, Gerber L, Moreira DM, McKeever M, et al. Smoking and risk of low- and high-grade prostate cancer: results from the REDUCE study. Clin Cancer Res 2014;20:5331-8.

34. Boudreau DM, Yu O, Johnson J. Statin use and cancer risk: a comprehensive review. Expert Opin Drug Saf 2010;9:603-21.

35. Pelton K, Freeman MR, Solomon KR. Cholesterol and prostate cancer. Curr Opin Pharmacol 2012;12:751-9.

36. Yu O, Eberg M, Benayoun S, Aprikian A, Batist G, Suissa S, et al. Use of statins and the risk of death in patients with prostate cancer. J Clin Oncol 2014;32:5-11.

37. National Cholesterol Education Program Expert Panel on Detection, Evaluation, and Treatment of High Blood Cholesterol in Adults. Third Report of the National Cholesterol Education Program (NCEP) Expert Panel on Detection, Evaluation, and Treatment of High Blood Cholesterol in Adults (Adult Treatment Panel III) final report. Circulation 2002; 106:3143-421.

38. Stone NJ, Robinson JG, Lichtenstein AH, Bairey Merz CN, Blum CB, Eckel $\mathrm{RH}$, et al. 2013 ACC/AHA guideline on the treatment of blood cholesterol to reduce atherosclerotic cardiovascular risk in adults: a report of the American College of Cardiology/American Heart Association Task Force on Practice Guidelines. J Am Coll Cardiol 2014;63:2889-934.

39. American Society of Clinical Oncology. The state of cancer care in America, 2014: a report by the American Society of Clinical Oncology. J Oncol Pract 2014;10:119-42. 


\section{Cancer Epidemiology, Biomarkers \& Prevention}

\section{Statin Use and Prostate Cancer Aggressiveness: Results from the Population-Based North Carolina-Louisiana Prostate Cancer Project}

Emma H. Allott, Laura Farnan, Susan E. Steck, et al.

Cancer Epidemiol Biomarkers Prev 2016;25:670-677. Published OnlineFirst January 27, 2016.

\section{Updated version Access the most recent version of this article at:} doi:10.1158/1055-9965.EPI-15-0631

Supplementary Access the most recent supplemental material at:

Material http://cebp.aacrjournals.org/content/suppl/2016/01/27/1055-9965.EPI-15-0631.DC1

Cited articles This article cites 38 articles, 8 of which you can access for free at: http://cebp.aacrjournals.org/content/25/4/670.full\#ref-list-1

E-mail alerts Sign up to receive free email-alerts related to this article or journal.

Reprints and To order reprints of this article or to subscribe to the journal, contact the AACR Publications Department Subscriptions at pubs@aacr.org.

Permissions To request permission to re-use all or part of this article, use this link http://cebp.aacrjournals.org/content/25/4/670.

Click on "Request Permissions" which will take you to the Copyright Clearance Center's (CCC) Rightslink site. 